\title{
Dipole properties of the upper rim phosphorylated calix[4]arenes in the Langmuir-Blodgett films
}

\author{
B.O. Nesterenko, Z.I. Kazantseva, O.A. Stadnyk \\ Institute of Semiconductor Physics of the National Academy of Sciences of Ukraine, Prospekt Nauky 45, \\ 03028, Kyiv-28, Ukraine, Phone: 380(44)265-60-40,E-mail: nesteren@lab32.kiev.ua
}

\section{De Rossi}

Centro 'E Piaggio' Facolta di Ingegneria Universita di Pisa, Via Dioticalvi, 2, 56100 Pisa, Italy Phone: 39(50)55-36-39, E-mail: derossi@piaggio.ccii.unipi.it

\section{V.I. Kalchenko}

Institute of Organic Chemistry of the National Academy of Sciences of Ukraine, Prospekt Zabolotnogo 160, 252 143, Kyiv, Ukraine, Phone: 380(44)559-60-67,E-mail:vik@ukrpak.net

\begin{abstract}
A$ - diagrams, ellipsometry and contact potential difference measurements were used to study the preparation and surface potential of calixarenes LBFs modified with phosphorylated groups at upper molecular rim (see text). The Langmuir nature of the obtained films on the silicon substrates has been proved. The surface potentials and molecular concentrations have been measured for all the compounds under investigation. Normal components of the dipole moments were analyzed for observed calixarenes. In monolayered structures they appeared to be positive for the molecules as a whole as well as for their parts.
\end{abstract}

Keywords: Langmuir-Blodgett films, calixarene, dipole moments.

Paper received 12.09.00; revised manuscript received 15.12.00; accepted for publication 16.02.01.

\section{Introduction}

Considerable recent attention has been focused on the calixarenes $\mathrm{C}[\mathrm{n}] \mathrm{A}$, which are used more and more widely [1-3]. Due to their basket-like shape they can adsorb and then keep molecules of different types. In other words «host-guest» complexes are created. This peculiarity is important for gas and liquid sensor application because it satisfies two main requirements - selectivity and sensitivity $[4,5]$.

The search of new materials is continued in order to extend the sensor performances abilities. Particularly, new calixarenes derivatives modified with different functional groups are synthesized. Such compounds are capable to link to definite molecules from gas (vapor) or liquid environments selectively [6,7]. From this point of view, the functional groups which contain phosphorus atoms seem to be attractive. Since phosphorus atoms can be in different oxidation states, they can tie the ions of transition metals, neutral proton-donor molecules and biological macromolecules (immobilization of proteins).

Obviously the adsorption properties of calixarenes are governed not only by the geometrical form but by their dipole nature as well. That is why in this work the dipole parameters were studied for Langmuir-Blodgett films (LBF) prepared using phosphorilated C[4]A derivatives.

\section{Experimental}

The new calixarenes (CA) compounds with different phosphorilated functional groups were synthesized in the Institute of Organic Chemistry of the National Academy of Sciences of Ukraine. Their molecular structures are shown in Fig. 1. One can see that compound I contains one functional group $O=P\left(O \mathrm{Pr}_{i}\right)_{2}\left(\operatorname{Pr}_{i}-\right.$ isoprophyl); II - with four such groups; III - the substituting group involved phenyl $(\mathrm{Ph})$ and isopropyl aggregates $O=$ $=P(O P r i) P h$. So far we shall indicate the samples of these 


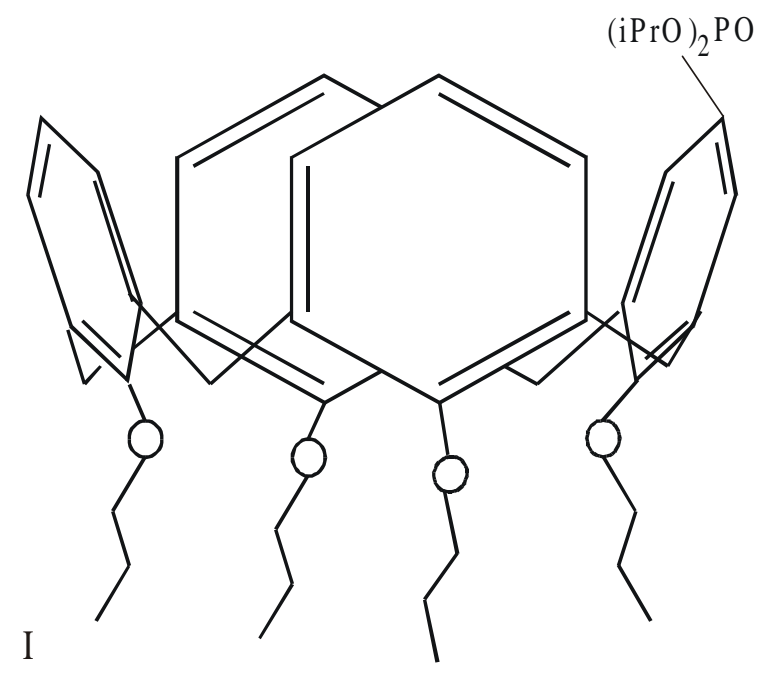

three types as I, II and III. The cone shape of molecules was determined with quantum-chemical calculations and nuclear magnetic resonance spectroscopy data [8]. The cone height without functional groups can be calculated using the sizes of crown size $(\sim 0.5 \mathrm{~nm})$ and length of the chain $\mathrm{C}_{3} \mathrm{H}_{5}(\sim 0.4 \mathrm{~nm})$. It appeared to be about $1 \mathrm{~nm}$.

The chloroform solution $0.5 \mathrm{mg} / \mathrm{ml}$ was used for spreading on the bidistilled water $(\mathrm{pH}=6.5)$ used as a surface.

Two types of the $\mathrm{Si}(100)$ substrate covered with the native oxide layer were used for the next LBF transfer $i$ ) silicon wafers boiled in the $\mathrm{HCl}: \mathrm{H}_{2} \mathrm{O}_{2}: \mathrm{H}_{2} \mathrm{O}$ mixture to retain a hydrophilic surface; ii) ones which were subsequently treated in the hexamethyldisilazan vapor for $30 \mathrm{~min}$ for hydrophobization. In all cases they had the dimensions $(25 \times 7 \times 0,5) \mathrm{mm}^{3}$.

LBFs were formed in the standard way of vertical transfer under control of the transfer coefficient and $\pi-A-$ diagrams $(\pi$-the pressure inside the organic film on the water surface, $A$ - the area per one molecule), Fig. 2.

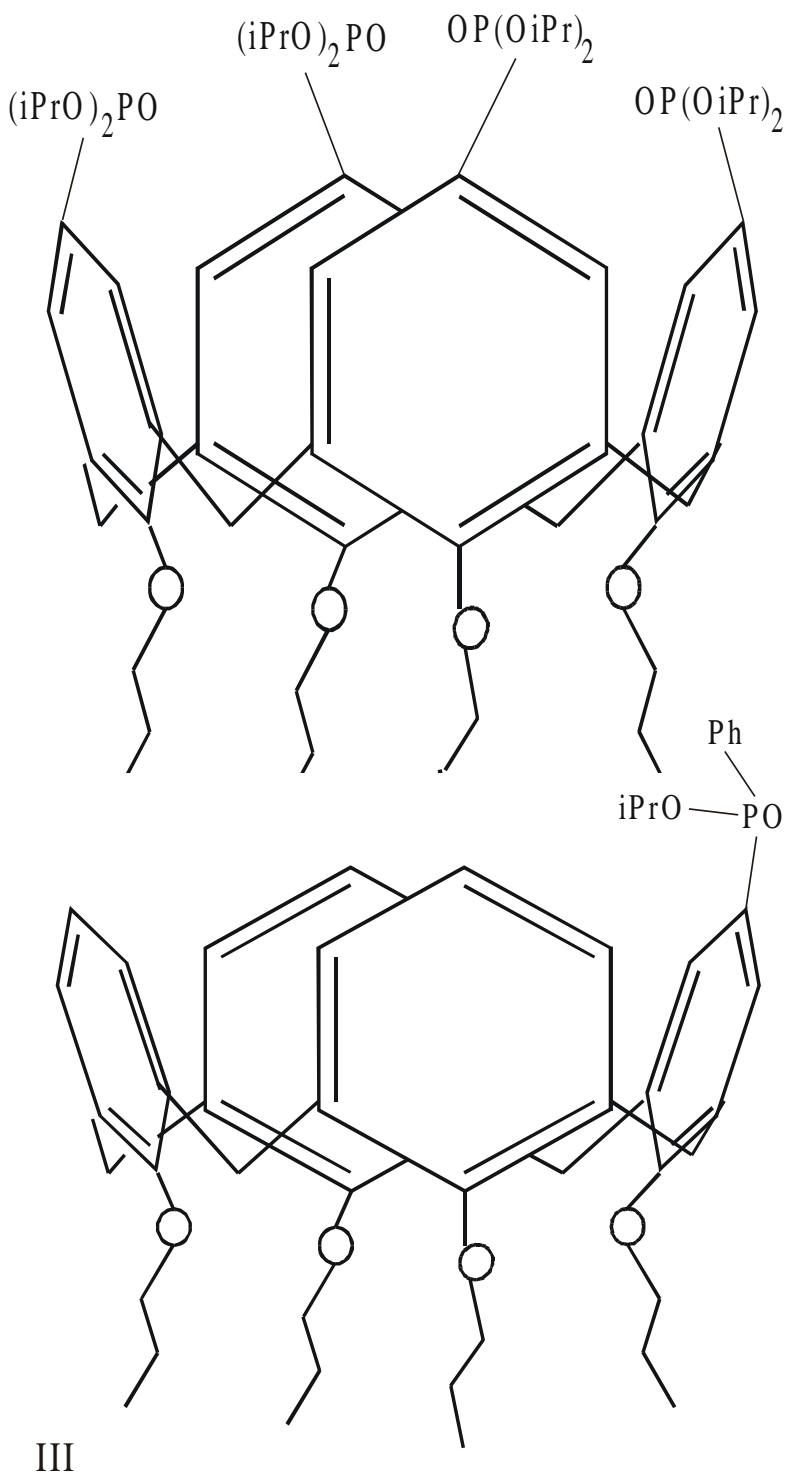
The LBFs were deposited at the surface tension of 18 $20 \mathrm{mN} / \mathrm{m}$ either by downwards and upwards strokes or by only upstrokes. In the latter case the downstrokes were made through the expanded monolayer. The dipping rate was $2 \mathrm{~mm} / \mathrm{min}$. The transfer pressures were determined experimentally. They corresponded to the maximal $\mathrm{p}$ value, at which the monolayer on the water surface remained stable. For the compounds of all three types the mono- and bilayered films were prepared on the hydrophilic and hydrophobic substrates correspondingly. The LBF thickness was measured ellipsometrically using a laser beam $\left(\lambda=0.6328 \mu \mathrm{m}\right.$; incidence angle, $\left.60^{\circ}\right)$. The reversed ellipsometric problem was solved numerically for the «substrate-optically transparent film» model.

Kelvin-probe method was used to study the surface potential. A gold reference electrode of round shape and $5 \mathrm{~mm}$ diameter was located at $0.03-0.05 \mathrm{~mm}$ from the sample and vibrated at a frequency of $385 \mathrm{~Hz}$. Contact potential differences between electrode and $\mathrm{LBF}\left(V_{\mathrm{LBF}}\right)$

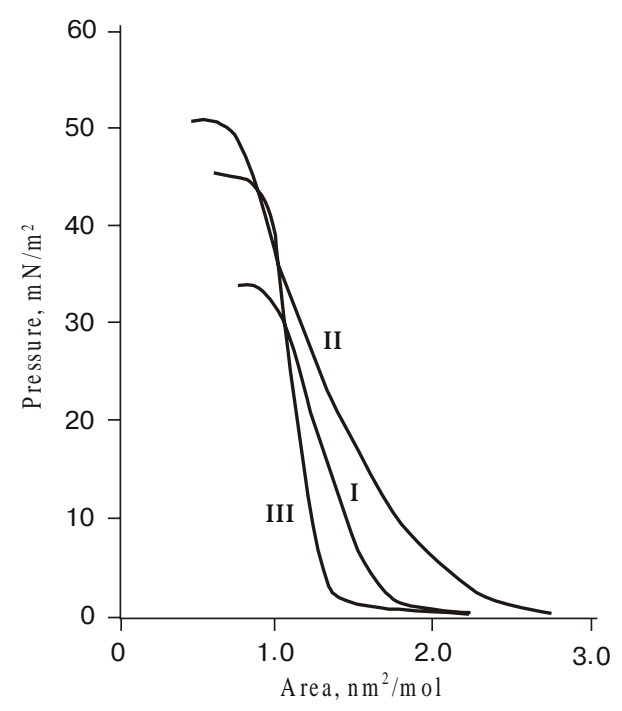

Fig.1. The schematic images of calixarene molecules I, II and III. 
or the substrate ( $\left.V_{\text {SUBST }}\right)$ were determined from the minimum positions at the dependencies of alternate signal $V_{\sim}$ on the compensating bias $V_{=}$. The synchronic detector was used in the minimum vicinity. It provided the scheme sensitivity not worse than $0,02 \mathrm{eV}$.

The Kelvin-probe method can give some information about the LBF's surface potential homogeneity and so about the structural defectiveness. Namely, the $V_{\sim}\left(V_{=}\right)$ curve rises steeply for uniform LBF and is less steep for the imperfect one. This feature was used for the qualitative characterization of LBF defectiveness (parameter $K$ ). The K-value was determined as the ratio $K=\left(V_{\sim \mathrm{LBF}}\right) /\left(V_{\sim}\right.$ SUBST $)$. Both $\left(V_{\sim \mathrm{LBF}}\right)$ and $\left(V_{\sim \text { SUBST }}\right)$ magnitudes were chosen to correspond the alternate signals $V_{\sim}$ at $V_{=}=$ $=0.2 \mathrm{~V}$ aside the $V_{\sim}\left(V_{=}\right)$- minimum on LBF and substrate, Fig. 3.

\section{Results and discussion}

The $\pi$ - $A$ - diagrams are shown in Fig. 2. Taking into account the rather flat character of these $\pi$ - $A$ dependencies we used for the definition at $A$-values for close packed layers the method of the tangent to the curve to zero pressure in condition of transfer. These data for compounds I, II and III are represented in Table 1. Ellipsometric measurments on LBFs gave the values of both reflactive index 1.45 and thickness $1.0 \pm 0.1 \mathrm{~nm}$ for monolayers I, II and III on the hydrophilic substrates. They increased by a factor 2 for bilayers on the hydrophobic substrates just in accordance with the transfer coefficient. This fact independently proves the Langmuir nature of the prepared films.

Surface potential measurements will be discussed for hydrophilic and hydrophobic substrates separately.

\section{Hydrophilic substrates, monomolecular LBFs}

Results for hydrophilic substrates are presented in Table 1. Following [9] we shall consider the changes which are induced by organic films into the substrates surface potential, $\Delta V=V_{\mathrm{LBF}}-V_{\mathrm{SUBST}}$. For all cases the $\Delta V-$ values appeared to be positive, $\Delta V>0$. It means that the substrate's work function decreases after the LBF transfer. The molecules in LBFs must contain dipoles with the positive normal component directed from the substrate $(-)$ toward the air $(+)$. When trying to understand such a

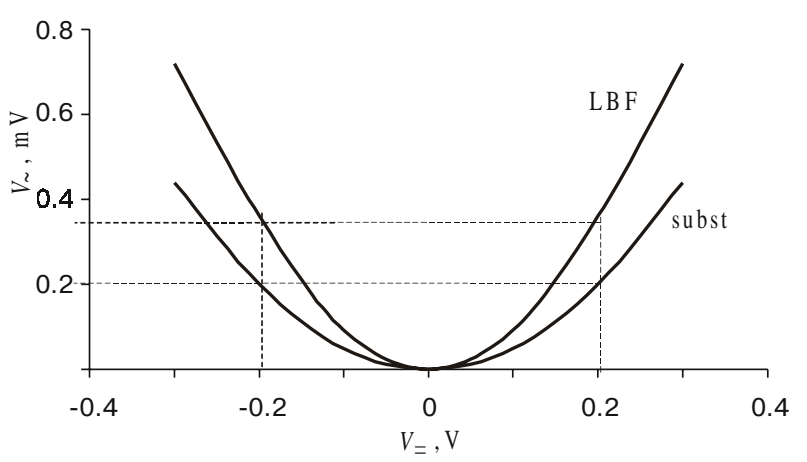

Fig. 3. The illustration of the defectness parameter $\mathrm{K}$ definition. The $\mathbf{V}_{\sim}\left(\mathbf{V}_{=}\right)$minimum positions of both LBF and substrate are brought into coincidence at $\mathbf{V}_{=}=0$ point for simplicity.

situation we have to take into account the complicated structure of molecules I, II and III which includes the macrocycle, $\operatorname{Pr}_{i}$ (or $\operatorname{Pr}_{i}-\mathrm{Ph}$ ) - groups and four $\mathrm{C}_{3} \mathrm{H}_{7}-$ chains, see Fig. 1. The latest ones are positioned on the outer side of the films and have the end $\mathrm{CH}_{3}-$ groups with the intrinsic positive dipoles $0.35 \mathrm{D}$ [9]. So first of all it was necessary to determine their maximal contribution to the measured $\Delta V$-values. The calculations for LBFs I, II and III were performed using the data of [9]. They gave the values $0.30 ; 0.26$ and $0.41 \mathrm{~V}$ respectively, which were evidently smaller than $\Delta V$ (see Table 1$)$. In reality the contribution of the end $\mathrm{CH}_{3}$ - dipoles should be even smaller due to the declination of their orientation from the surface normal [10].

It is impossible to explain the $\Delta V$ experimental results by the presence of $\mathrm{C}_{3} \mathrm{H}_{7}-$ chains only. It becomes evident that in compounds I, II and III the molecular macrocycles with joint to them $\mathrm{Pr}-$ and $\mathrm{Ph}$ - groups have resultant positive moments as well.

To elucidate this matter and to possibly quantify the observed effects a simplifying assumption will be done. In a first approximation we shall suppose that $\mathrm{Pr}-$ and $\mathrm{Ph}$ - groups do not change the dipolar properties of the rest part of the molecule (macrocycle + four $\mathrm{C}_{3} \mathrm{H}_{7}-$ chains). Then the total dipole moments of compounds I and II must differ due to the number of $\operatorname{Pr}_{i}$ - groups only. Namely, molecule I contain one such group, II - four group (see Fig. 1). For these LBFs one could write the following equations

Table 1. The experimental data for LBFs I, II and III, one monolayer, the hydrophilic substrate.

\begin{tabular}{cccccccc}
\hline Compound & $A, \mathrm{~nm}^{2}$ & $N, 10^{13} \mathrm{~cm}^{-2}$ & $\Delta V, \mathrm{~V}$ & $\Delta \mu, \mathrm{D}$ & $\Delta \mu_{\mathrm{Pri}(\mathrm{Ph}), \mathrm{D}}$ & $\mu, \mathrm{D}$ & $K$, a.u. \\
\hline I & $1.75 \pm 0.05$ & 5.71 & $0.37 \pm 0.05$ & 1.20 & 0.51 & 1.71 & 1.0 \\
\hline II & $2.07 \pm 0.05$ & 4.83 & $0.60 \pm 0.05$ & 1.20 & $4 \times 0.51=2.04$ & 3.24 & 1.4 \\
\hline III & $1.30 \pm 0.05$ & 7.69 & $0.72 \pm 0.05$ & 1.20 & 1.28 & 2.48 & 2.3 \\
\hline \hline
\end{tabular}


B.O. Nesterenko et al.: Dipole Properties of the upper rim phosphorylated ...

$\Delta V_{\mathrm{I}}=\left(4 \Delta \mu_{\mathrm{CH}_{3}}+\Delta \mu_{\mathrm{MACRO}}+1 \Delta \mu_{\mathrm{Pri}}\right) N_{\mathrm{I}}$

$\Delta V_{\mathrm{II}}=\left(4 \Delta \mu_{\mathrm{CH}_{3}}+\Delta \mu_{\mathrm{MACRO}}+1 \Delta \mu_{\mathrm{Pri}}\right) N_{\mathrm{II}}$

Here the terms in parentheses correspond to the normal components of dipoles which belong to $\mathrm{C}_{3} \mathrm{H}_{7}$ - chains $\left(4 \Delta \mu_{\mathrm{CH}_{3}}\right)$, macrocycle $\left(\Delta \mu_{\mathrm{MACRO}}\right)$ and joint phosphorilated group Pri $\left(\Delta \mu_{\mathrm{Pri}}\right), N_{\mathrm{I}, \mathrm{II}}=1 / A_{\mathrm{I}, \mathrm{II}}-$ concentration of molecules in LBF.

At present the orientations of $\mathrm{C}_{3} \mathrm{H}_{7}$ - chains are not known. Thus two equations (1) contain three unknowns ( $4 \Delta \mu_{\mathrm{CH}_{3}}, \Delta \mu_{\text {MACRO }}, \Delta \mu_{\mathrm{Pri}}$ ) and cannot be solved. After combining $4 \Delta \mu_{\mathrm{CH}_{3}}$ and $\Delta \mu_{\mathrm{MACRO}}, \Delta \mu=4 \Delta \mu_{\mathrm{CH}_{3}}+$ $+\Delta \mu_{\mathrm{MACRO}}$ one can get the system with two unknowns $\left(\Delta \mu, \Delta \mu_{\text {Pri }}\right)$

$$
\begin{aligned}
& \Delta V_{\mathrm{I}}=\left(\Delta \mu+1 \Delta \mu_{\mathrm{Pri}}\right) N_{\mathrm{I}} \\
& \Delta V_{\mathrm{II}}=\left(\Delta \mu+4 \Delta \mu_{\mathrm{Pri}}\right) N_{\mathrm{II}}
\end{aligned}
$$

The system was used to calculate dipole moments $\Delta \mu$, $\Delta \mu_{\text {Pri }}$ when inserting the experimental values $\Delta V_{\mathrm{I}, \mathrm{II}}$ and $N_{\mathrm{I}, \mathrm{II}}$. Then, using the equation $\Delta V_{\mathrm{III}}=\left(\Delta \mu+\Delta \mu_{\mathrm{Ph}}\right) N_{\mathrm{III}}$ the component $\Delta \mu_{\mathrm{Ph}}$ was determined for LBF III. All these data are presented in Table 1 as well as the magnitudes of total dipole moments $\mu$ for molecules I, II and III which were obtained from $(\Delta V, A)$ - results in a direct way without any assumptions. All of them appear to be positive.

One can expect the existence of dipole moments $\Delta \mu_{\text {Pri, }}$, $\Delta \mu_{\mathrm{Ph}}>0$ in LBFs on the hydrophilic substrate from general considerations. Actually Pri - and $\mathrm{Ph}-$ groups in the phosphorilated calixarenes contain atoms of phosphorus, oxygen and $\mathrm{C}_{3} \mathrm{H}_{7}-$ groups. The shift of electron density takes place to the oxygen atoms, which positions are close to the substrate. Thus the dipole formation is promoted. The end $\mathrm{CH}_{3}$ - dipoles of $\mathrm{C}_{3} \mathrm{H}_{7}-$ chains bring the additional contribution. As a result the $\Delta \mu_{\mathrm{Pri}}$ and $\Delta \mu_{\mathrm{Ph}}$ became relatively large (see Table 1).

So the investigations of the surface potential on the LBFs I, II and III have shown that total molecular dipole moments are positive and their magnitudes depend on the type of compound. The most polar film appears to be II, which has four substituting phosphoric groups $\mathrm{P}(\mathrm{O})(\mathrm{OPri})_{2}$. However, the dipole moment of the separate functional group appeared to be the gteatest at substance III. It is equal to $1.28 \mathrm{D}$.

The obtained values $\mu, \Delta \mu, \Delta \mu_{\mathrm{Pri}}$ and $\Delta \mu_{\mathrm{Ph}}$ can be influenced by the LBF's defectiveness. The structural breaches create in LBF regions with changed molecular orientation. Surface potential inhomogeneity arises. The analysis of $V_{\sim}\left(V_{=}\right)$curves in Kelvin method indicates the LBF's I, II and III homogeneity to be better than the substrates' one $\left(K_{\mathrm{I}} \sim 1 ; K_{\mathrm{II}} \sim 1.4 ; K_{\mathrm{III}} \sim 2,3\right.$, see Table1). A possible reason of this effect could be large molecules' sizes $\left(A=1.30-2.07 \mathrm{~nm}^{2}\right)$ and strong lateral interactions in $\mathrm{LBF}$. As a result the mentioned organic layers
Table 2. The data for bilayers I, II, III and stearic acid, the hydrophobic substrate.

\begin{tabular}{ccc}
\hline \hline Compound & \multicolumn{1}{c}{$\Delta V, \mathrm{~V}$} & $K$, a.u. \\
\hline I & $0.22 \pm 0.05$ & 2.0 \\
\hline II & $0 \pm 0.05$ & 0.4 \\
\hline III & $0.50 \pm 0.05$ & 0.4 \\
\hline Stearic acid & $0.02 \pm 0.05$ & 1.0 \\
\hline \hline
\end{tabular}

smooth the initial substrates' potential relief. For comparison we performed experiments on a model object perfect bilayers of stearic acid. They have demonstrated substrate potential relief, $K \sim 1$. Probably it takes place due to the small molecule's cross section $\left(0.2 \mathrm{~nm}^{2}\right)$ of stearic acid, which repeat the substrate relief.

\section{Hydrophobic substrates, bilayered LBFs}

Table 2 reports the experimental data $(\Delta V, K)$ for bilayered films I, II and III. The same information for stearic acid bilayer as a model object is presented as well. In the latter case the potential change $\Delta V$ appeared to be positive and very small $(\Delta V=0.02-0.05 \mathrm{~V}$ for different samples). One can explain it by the mutual dipole compensation of upper and lower monolayers and partial screening of lower layer $\mathrm{CH}_{3}$ - dipoles by the substrate mobile charge carriers.

For bilayered films I, II and III the $\Delta \mathrm{V}$-values were much smaller than that for monolayers (compare the data of Tables 1 and 2). Apparently there is also dipole compensation in these LBFs. However its degree is different for compounds I, II and III, being the lowest for the sample III. One of the possible explanations of these phenomena could be strong disordering of Pri-( $\mathrm{Ph}-)$ dipoles. This effect must be larger for LBF III because of the much larger Ph-moment $\Delta \mu_{\mathrm{Ph}}$ compared to the $\Delta \mu_{\mathrm{Pri}}-$ value (by factor of $\sim 2.5$, see Table 1 ).

The above considerations qualitatively agree with defectiveness characteristic $K$ too. For bilayers I, II and III the inequality $K_{\mathrm{I}}>K_{\mathrm{II}, \mathrm{III}}$ was obtained (see Table 2 ). The dipole-dipole repulsive interaction of Pri-groups must be the weakest in LBF I which have only one such group per molecule. In LBF II the amount of Pri-dipoles is four times larger. Thus induced inhomogeneity increases. This effect is maximal in film III due to the large dipole moment $\Delta \mu_{\mathrm{Ph}}$.

\section{Conclusions}

The preparation and surface potentials were studied for Langmuir-Blodgett films of upper rim phosphorylated calix[4]arenes when using $\pi$ - $A$ - diagrams, ellipsometry and Kelvin-probe method. It has been established that monomolecular layers lower considerably the hydrophilic substrate's work function. The sign and magnitudes of 


\section{B.O. Nesterenko et al.: Dipole Properties of the upper rim phosphorylated ...}

dipoles were estimated for phosphorylated Pri and $\mathrm{Ph}$ groups linked to the calixarene macrocycles. It was revealed that there is dipole-dipole partial compensation only in bilayers I, II and III on the hydrophobic substrates. Possible reasons explaining these phenomena were discussed. One of them is strong dipole repulsion of the phosphorylated Pri and Ph groups.

\section{Acknowledgments}

This work was performed in the framework of grant INTAS-Ukraine N 95-0129 and with its financial assistance. The authors highly appreciate the INTAS support.

\section{References}

1. J. Vicens, V. Bohmer. Calixarenes, a Versatile Class of Macrocyclic Compounds, Eds. Kluwer, Dordrecht, (1991).

2. C. D. Gutsche. Calixarenes. Royal Society of Chemistry, Cambridge (1989)

3. F.L. Dickert, O. Schuster, Supramolecular detection of solvent vapours with calixarenes: mass-sensitive sensors, molecular mechanics and BET studies // Mikrochim. Acta 119, pp. 55-62 (1995).
4. Z. Ali-Adid, F. Davis, P. Hodge, C.J. Stirling, Structures and binding of LB films of calix-8-arenes // Supramol. Sci. 4, pp. 201-206 (1997).

5. J. Lipkowski, O.I. Kalchenko, J. Slowikovska, V.I. Kalchenko, O.V. Lukin, L.N. Markovsky, R.Nowakowski, Host-guest interaction of calix[4]resorcinarenes with benzene derivatives in conditions of reversed-phase high-performance liquid chromatography. Determination of stability constants // J . Org. Chem. 11, pp. 426-435 (1998).

6. P. Nelli, E. Dalcanale, G. Faglia, G. Sberveglieri, P. Soncini, Cavitands as selective materials for QMB sensors for nitrobenzene and other aromatic vapours // Sensors and Actuators B, 13-23, pp. 302-304 (1993).

7. S. Rosler, R. Lucklum, R. Borngraber, J. Hartmann, P. Hauptmann, Sensor system for detection of organic pollutants in water by thickness shear mode resonators // Sensors and Actuators B 48 pp. 415-423, (1998).

8. I. Neda, T. Kaukorat, R. Schmutzler, Functionalization of the periphery of calix[n]resorcinarenes with phosphorus-containing substituents // Main Group Chemistry News. 6, pp. 4-23 (1998).

9. V. Vogel, D. Mobius, Hydrated polar groups in lipid monolayers: effective local dipole moments and dielectric properties // Thin Solid Films, 159, pp. 73-81 (1988).

10. A.V. Nabok, N.V. Lavrik, Z.I. Kazantseva, B.A. Nesterenko, V.I. Kalchenko, L.N. Markovskiy, Complexing properties of calix[4]resorcinolarene LB films // Thin Solid Films, 259, pp. 244-247 (1995). 